\title{
Shimura curve computations via K3 surfaces of Néron-Severi rank at least 19
}

\author{
Noam D. Elkies \\ Department of Mathematics, Harvard University, Cambridge, MA 02138 \\ elkies@math.harvard.edu \\ Supported in part by NSF grant DMS-0501029
}

\section{Introduction}

In E1 we introduced several computational challenges concerning Shimura curves, and some techniques to partly address them. The challenges are: obtain explicit equations for Shimura curves and natural maps between them; determine a Schwarzian equation on each curve (a.k.a. Picard-Fuchs equation, a linear second-order differential equation with a basis of solutions whose ratio inverts the quotient map from the upper half-plane to the curve); and locate CM (complex multiplication) points on the curves. We identified some curves, maps, and Schwarzian equations using the maps' ramification behavior; located some CM points as images of fixed points of involutions; and conjecturally computed others by numerically solving the Schwarzian equations.

But these approaches are limited in several ways: we must start with a Shimura curve with very few elliptic points (not many more than the minimum of three); maps of high degree are hard to recover from their ramification behavior, limiting the range of provable CM coordinates; and these methods give no access to the abelian varieties with quaternionic multiplication (QM) parametrized by Shimura curves. Other approaches somewhat extend the range where our challenges can be met. Detailed theoretical knowledge of the arithmetic of Shimura curves makes it possible to identify some such curves of genus at most 2 far beyond the range of [E1] (see e.g. Rob|GR]), though not their Schwarzian equations or CM points. Roberts Rob showed in principle how to find $\mathrm{CM}$ coordinates using product formulas analogous to those of $\mathrm{GZ}$ for differences between CM $j$-invariants, but such formulas have yet to be used to verify and extend the tables of [E1]. Errthum [Er] recently used Borcherds products to verify all the conjectural rational coordinates for CM points tabulated in E1 for the curves associated to the quaternion algebras over $\mathbf{Q}$ ramified at $\{2,3\}$ and $\{2,5\}$; it is not yet clear how readily this technique might extend to more complicated Shimura curves. The $p$-adic numerical techniques of [E3 give access to further maps and CM points. Finally, in the $\{2,3\}$ and $\{2,5\}$ cases Hashimoto and Murabayashi had already parametrized the relevant QM abelian surfaces in $1995[\mathrm{HM}]$, but apparently such computations have not been pushed further since then.

In this paper we introduce a new approach, which exploits the fact that some Shimura curves also parametrize K3 surfaces of Néron-Severi rank at least 19. 
"Singular" K3 surfaces, those whose Néron-Severi rank attains the characteristiczero maximum of 20 , then correspond to CM points on the curve. We first encountered such parametrizations while searching for elliptic K3 surfaces of maximal Mordell-Weil rank over $\mathbf{Q}(t)$ (see E4]), for which we used the K3 surface corresponding to a rational non-CM point on the Shimura curve $\mathrm{X}(6,79) /\left\langle w_{6.79}\right\rangle$ of genus 2 . The feasibility of this computation suggested that such parametrizations might be used systematically in Shimura curve computations.

This approach is limited to Shimura curves associated to quaternion algebras over Q. Within that important special case, though, we can compute curves and CM points that were previously far beyond reach. The periods of the K3 surfaces should also allow the computation of Schwarzian equations as in [LY], though we have not attempted this yet. We do, however, find the corresponding QM surfaces using Kumar's recent formulas $\mathrm{Ku}$ that make explicit Dolgachev's correspondence Do between Jacobians of genus-2 curves and certain K3 surfaces of rank at least 17. The parametrizations do get harder as the level of the Shimura curve grows, but it is still much easier to parametrize the K3 surfaces than to work directly with the QM abelian varieties - apparently because the level, reflected in the discriminant of the Néron-Severi group, is spread over 19 NéronSeveri generators rather than the handful of generators of the endomorphism ring 11 In this paper we illustrate this with several examples of such computations for the curves $\mathrm{X}(N, 1)$ and their quotients. As the example of $\mathrm{X}(6,79) /\left\langle w_{6 \cdot 79}\right\rangle$ shows, the technique also applies to Shimura curves not covered by $\mathrm{X}(N, 1)$, but already for $\mathrm{X}(N, 1)$ there is so much new data that we can only offer a small sample here: the full set of results can be made available online but is much too large for conventional publication. Since we shall not work with $\mathrm{X}(N, M)$ for $M>1$, we abbreviate the usual notation $\mathrm{X}(N, 1)$ to $\mathrm{X}(N)$ here.

The rest of this paper is organized as follows. In the next section, we review the necessary background, drawn mostly from [Vi|Rot2 BHPV], concerning Shimura curves, the abelian and K3 surfaces that they parametrize, and the structure of elliptic $\mathrm{K} 3$ surfaces in characteristic zero; then give $\mathrm{A}$. $\mathrm{Ku}-$ mar's explicit formulas for Dolgachev's correspondence, which we use to recover Clebsch-Igusa coordinates for QM Jacobians from our K3 parametrizations; and finally describe some of our techniques for computing such parametrizations. In the remaining sections we illustrate these techniques in the four cases $N=6$, $N=14, N=57$, and $N=206$. For $N=6$ we find explicit elliptic models for our family of $\mathrm{K} 3$ surfaces $S$ parametrized by $\mathrm{X}(6) /\left\langle w_{6}\right\rangle$, locate a few CM points to find the double cover $\mathrm{X}(6) \rightarrow \mathrm{X}(6) /\left\langle w_{6}\right\rangle$, transform $S$ to find an elliptic model with essential lattice $N_{\text {ess }} \supset E_{7} \oplus E_{8}$ to which we can apply Kumar's formulas, and verify that our results are consistent with previous computations of CM points [E1] and Clebsch-Igusa coordinates [HM]. For $N=14$ we exhibit $S$ and verify the location of a CM point that we computed numerically in [E1] but could not prove using the techniques of [E1E3]. For $N=57$, the first case for

\footnotetext{
1 It would be interesting to quantify the computational complexity of such computations in terms of the level and the CM discriminant; we have not attempted such an analysis.
} 
which $\mathrm{X}(N) /\left\langle w_{N}\right\rangle$ has positive genus, we exhibit the K3 surfaces parametrized by this curve, and locate all its rational CM points. For $N=206$, the last case for which $\mathrm{X}(N) /\left\langle w_{N}\right\rangle$ has genus zero, we exhibit the corresponding family of K3 surfaces and the hyperelliptic curves $\mathrm{X}(206)$ and $\mathrm{X}(206) /\left\langle w_{2}\right\rangle, \mathrm{X}(206) /\left\langle w_{103}\right\rangle$ covering the rational curves $\mathrm{X}(206) /\left\langle w_{206}\right\rangle$ and $\mathrm{X}(206) /\left\langle w_{2}, w_{103}\right\rangle$.

\section{Definitions and techniques}

Quaternion algebras over Q, Shimura curves, and QM abelian surfaces. Fix a squarefree integer $N>0$ with an even number of prime factors. There is then a unique indefinite quaternion algebra $A / Q$ whose finite ramified primes are precisely the factors of $N$. Let $\mathcal{O}$ be a maximal order in A. Since A is indefinite, all maximal orders are conjugate in $A$, and conjugate orders will be equivalent for our purposes. Let $\mathcal{O}_{1}^{*}$ be the group of units of reduced norm 1 in $\mathcal{O}$; let $\Gamma$ be the arithmetic subgroup $\mathcal{O}_{1}^{*} /\{ \pm 1\}$ of $A^{*} / \mathbf{Q}^{*}$; and let $\Gamma^{*}$ be the normalizer of $\Gamma$ in the positive-norm subgroup of $\mathrm{A}^{*} / \mathbf{Q}^{*}$. If $N=1$ then $\Gamma^{*}=\Gamma$; otherwise $\Gamma^{*} / \Gamma$ is an abelian group of exponent 2, and for each factor $d \mid N$ there is a unique element $w_{d} \in \Gamma^{*} / \Gamma$ whose lifts to $\mathrm{A}^{*}$ have reduced norms in $d \cdot \mathbf{Q}^{* 2}$.

Because $\mathrm{A}$ is indefinite, $\mathbf{A} \otimes_{\mathbf{Q}} \mathbf{R}$ is isomorphic with the matrix algebra $M_{2}(\mathbf{R})$, so the positive-norm subgroup of $\mathrm{A}^{*} / \mathbf{Q}^{*}$ is contained in $\mathrm{PSL}_{2}(\mathbf{R})$ and acts on the upper half-plane $\mathcal{H}$. The quotient $\mathcal{H} / \Gamma$ is then a complex model of the Shimura curve associated to $\Gamma$, usually called $\mathrm{X}(N, 1)$. In [E1] we called this curve $\mathcal{X}(1)$ in analogy with the classical modular curve $\mathrm{X}(1)$ (see below), since $N$ was fixed and we studied Shimura curves that we called $\mathcal{X}_{0}(p), \mathcal{X}_{1}(p)$, etc., associated with various congruence subgroups of $\mathrm{A}^{*} / \mathbf{Q}^{*}$. In this paper we restrict attention to $\mathcal{H} / \Gamma$ and its quotients by subgroups of $\Gamma^{*} / \Gamma$; thus we return to the usual notation, but simplify it to $\mathrm{X}(N)$ because we do not need $\mathrm{X}(N, M)$ for $M>1$. If $N=1$ then $\mathbf{A} \cong M_{2}(\mathbf{Q})$, and we may take $\mathcal{O}=M_{2}(\mathbf{Z})$, when $\Gamma=\Gamma^{*}=\mathrm{PSL}_{2}(\mathbf{Z})$ and $\mathcal{H}$ must be extended by its rational cusps before we can identify $\mathcal{H} / \Gamma$ with $\mathrm{X}(1)$. Here we study curves $\mathrm{X}(N)$ and their quotients only for $N>1$, and these curves have no cusps.

The Shimura curve $\mathrm{X}(N)$ associated to a quaternion algebra over $\mathbf{Q}$ has a reasonably simple moduli description. Fix a positive anti-involution $\varrho$ of A of the form $\varrho(\beta)=\mu^{-1} \bar{\beta} \mu$ for some $\mu \in \mathcal{O}$ with $\mu^{2}+N=0$. Then $\mathrm{X}(N)$ parametrizes pairs $(A, \iota)$ where $A$ is a principally polarized abelian surface and $\iota$ is an embedding of $\mathcal{O}$ into the $\operatorname{ring} \operatorname{End}(A)$ of endomorphisms of $A$, such that the Rosati involution is given by $\varrho$. See [Rot2, $\S 2$ and Prop. 4.1]. This gives $\mathrm{X}(N)$ the structure of an algebraic curve over $\mathbf{Q}$.

An abelian surface with an action of a (not necessarily maximal) order in a quaternion algebra is said to have "quaternionic multiplication" (QM). A complex multiplication $(\mathrm{CM})$ point of $\mathrm{X}(N)$ is a point, necessarily defined over $\overline{\mathbf{Q}}$, for which $A$ has complex multiplication, i.e. is isogenous with the square of a CM elliptic curve. We shall use the QM abelian surfaces $A$ to find models for the Shimura curves $\mathrm{X}(N)$ and locate some of their CM points. 
When $N=1$, an abelian surface together with an action of $\mathcal{O} \cong M_{2}(\mathbf{Z})$ is just the square of an elliptic curve, so we recover the classical modular curve $\mathrm{X}(1)$. We henceforth fix $N>1$. Then the group $\Gamma^{*} / \Gamma$, acting on $\mathrm{X}(N)$ by involutions that we also call $w_{d}$, is nontrivial. These involutions are again defined over $\mathbf{Q}$, taking $(A, \iota)$ to $\left(A_{d}, \iota_{d}\right)$ for some $A_{d}$ isogenous with $A$. Specifically, $A_{d}$ is the quotient of $A$ by the subgroup of the $d$-torsion group $A[d]$ annihilated by the two-sided ideal of $\mathcal{O}$ consisting of elements whose norm is divisible by $d$, and the principal polarization on $A_{d}$ is $1 / d$ times the pull-back of the principal polarization on $A$. In particular $A_{d}$ is $\mathrm{CM}$ if and only if $A$ is. Hence the notion of a $\mathrm{CM}$ point makes sense on the quotient of $\mathrm{X}(N)$ by $\Gamma^{*} / \Gamma$ or by any subgroup of $\Gamma^{*} / \Gamma$. If a CM point of discriminant $-D$ on $\mathrm{X}(N) /\left(\Gamma^{*} / \Gamma\right)$ is rational then the class group of $\mathbf{Q}(\sqrt{-D})$ must be generated by the classes of primes lying over factors $p \mid D$ that also divide $N$. Thus the class group has exponent 1 or 2 and bounded size; in particular, only finitely many $D$ can arise. In each of the cases $N=6,14,57$, and 206 that we treat in this paper, $N$ has two prime factors, so the class number is at most 4 and we can cite Arno $\mathrm{Ar}$ to prove that a list of discriminants of rational CM points is complete. When $N$ has 4 or 6 prime factors we can use Watkins' solution of the class number problem up to 100 Wa.

We have $A_{N} \cong A$ as principally polarized abelian surfaces, but for $N>1$ the embeddings $\iota, \iota_{N}$ are not equivalent for generic QM surfaces $A$. When we pass from $A$ to its Kummer surface we shall lose the distinction between $\iota$ and $\iota_{N}$, and so will at first obtain only the quotient curve $\mathrm{X}(N) /\left\langle w_{N}\right\rangle$. We shall determine its double cover $\mathrm{X}(N)$ by locating the branch points, which are the CM points on $\mathrm{X}(N) /\left\langle w_{N}\right\rangle$ for which $A$ is isomorphic to the product of two elliptic curves with CM by the quadratic imaginary order of discriminant $-N$ or $-4 N$; the arithmetic behavior of other CM points will then pin down the cover, including the right quadratic twist over $\mathbf{Q}$.

An abelian surface with QM by $\mathcal{O}$ has at least one principal polarization, and the number of principal polarizations of a generic surface with QM by $\mathcal{O}$ was computed in Rot1, Theorem 1.4 and $\S 6$ ] in terms of the class number of $\mathbf{Q}(\sqrt{-N})$. Each of these yields a map from $\mathrm{X}(N) /\left\langle w_{N}\right\rangle$ to $\mathcal{A}_{2}$, the moduli threefold of principally polarized abelian surfaces. This map is either generically $1: 1$ or generically $2: 1$, and in the $2: 1$ case it factors through an involution $w_{d}=w_{d^{\prime}}$ on $\mathrm{X}(N) /\left\langle w_{N}\right\rangle$ where $d, d^{\prime}>1$ are integers such that $N=d d^{\prime}$ and

$$
\mathrm{A} \cong\left(\frac{-N, d}{\mathbf{Q}}\right)\left[=\left(\frac{d, d^{\prime}}{\mathbf{Q}}\right)\right]
$$

(See the last paragraph of [Rot2, §4], which also notes that a $2: 1$ map occurs for $N=6$ and $N=10$, each of which has a unique choice of polarization. In the other cases $N=14,57,206$ that we study in this paper, only 1 : 1 maps arise, because the criterion (11) is not satisfied.) We aim to determine at least one of the maps $\mathrm{X}(N) /\left\langle w_{N}\right\rangle \rightarrow \mathcal{A}_{2}$ in terms of the Clebsch-Igusa coordinates on $\mathcal{A}_{2}$, 
and thus to find the moduli of the generic abelian surface with endomorphisms by $\mathcal{O} 2$

K3 surfaces, elliptic K3 surfaces, and the Dolgachev-Kumar correspondence. Let $F$ be a field of characteristic zero. Recall that a $K 3$ surface over $F$ is a smooth, complete, simply connected algebraic surface $S / F$ with trivial canonical class. The Néron-Severi group $\mathrm{NS}(S)=\mathrm{NS}_{\bar{F}}(S)$ is the group of divisors on $S$ defined over the algebraic closure $\bar{F}$, modulo algebraic equivalence. For a K3 surface this is a free abelian group whose rank, the Picard number $\rho=\rho(S)$, is in $\{1,2,3, \ldots, 20\}$. The intersection pairing gives $\operatorname{NS}(S)$ the structure of an integral lattice; by the index theorem for surfaces, this lattice has signature $(1, \rho-1)$, and for a K3 surface the lattice is even: $v \cdot v \equiv 0 \bmod 2$ for all $v \in \mathrm{NS}(S)$. Over $\mathbf{C}$, the cycle class map embeds $\mathrm{NS}(S)$ into the "K3 lattice" $H^{2}(S, \mathbf{Z}) \cong \mathrm{II}_{3,19} \cong U^{3} \oplus E_{8}\langle-1\rangle^{2}$, where $U=\mathrm{II}_{1,1}$ is the "hyperbolic plane" (the indefinite rank-2 lattice with Gram matrix $\left(\begin{array}{ll}0 & 1 \\ 1 & 0\end{array}\right)$ ), and $E_{8}\langle-1\rangle$ is the $E_{8}$ root lattice made negative-definite by multiplying the inner product by -1 . The Torelli theorem of Piateckii-Shapiro and Šafarevič [PSS] describes the moduli of $\mathrm{K} 3$ surfaces, at least over $\mathbf{C}$ : the embedding of $\mathrm{NS}(S)$ into $\mathrm{II}_{3,19}$ is primitive, that is, realizes $\mathrm{NS}(S)$ as the intersection of $\mathrm{II}_{3,19}$ with a $\mathbf{Q}$-vector subspace of $\mathrm{II}_{3,19} \otimes \mathbf{Q}$; for every such lattice $L$ of signature $(1, \rho-1)$, there is a nonempty (coarse) moduli space of pairs $(S, \iota)$, where $\iota: L \rightarrow \mathrm{NS}(S)$ is a primitive embedding consistent with the intersection pairing; and each component of the moduli space has dimension $20-\rho$. Moreover, for $\rho=20,19,18,17$ these moduli spaces repeat some more familiar ones: isogenous pairs of CM elliptic curves for $\rho=20$, elliptic and Shimura modular curves for $\rho=19$, moduli of abelian surfaces with real multiplication or isogenous to products of two elliptic curves for $\rho=18$, and moduli of abelian surfaces for certain cases of $\rho=17$. Note the consequence that an algebraic family of K3 surfaces in characteristic zero with $\rho \geq 19$ whose members are not all $\bar{F}$-isomorphic must have $\rho=19$ generically, else there would be a positive-dimensional family of K3 surfaces with $\rho \geq 20$.

An elliptic K3 surface $S / F$ is a $\mathrm{K} 3$ surface together with a rational map $t: S \rightarrow \mathbf{P}^{1}$, defined over $F$, whose generic fiber is an elliptic curve. The classes of the zero-section $s_{0}$ and fiber $f$ in $\operatorname{NS}(S)$ then satisfy $s_{0} \cdot s_{0}=-2, s_{0} \cdot f=1$, and $f \cdot f=0$, and thus generate a copy of $U$ in $\operatorname{NS}(S)$ defined over $F$. Conversely, any copy of $U$ in $\operatorname{NS}(S)$ defined over $F$ yields a model of $S$ as an elliptic surface: one of the standard isotropic generators or its negative is effective, and has 2 independent sections, whose ratio gives the desired map to $\mathbf{P}^{1}$. We often use this construction to transform one elliptic model of $S$ to another that would be harder to compute directly. (Warning: in general one might have to subtract some base locus from the effective generator to recover the fiber class $f$.)

Since $\operatorname{disc}(U)=-1$ is invertible, we have $\operatorname{NS}(S)=\left\langle s_{0}, f\right\rangle \oplus\left\langle s_{0}, f\right\rangle^{\perp}$, with the orthogonal complement $\left\langle s_{0}, f\right\rangle^{\perp}$ having signature $(0, \rho-2)$; we thus write $\left\langle s_{0}, f\right\rangle^{\perp}=N_{\text {ess }}\langle-1\rangle$ for some positive-definite even lattice $N_{\text {ess }}$, the "essential

\footnotetext{
2 Alas we cannot say simply "find the generic abelian surface with endomorphisms by $\mathcal{O}$ ", even up to quadratic twist, because there are abelian surfaces with rational moduli but no model over $\mathbf{Q}$.
} 
lattice" of the elliptic K3 surface. A vector $v \in N_{\text {ess }}$ of norm 2, corresponding to $v \in\left\langle s_{0}, f\right\rangle^{\perp}$ with $v \cdot v=-2$, is called a "root" of $N_{\text {ess }}$; let $R \subseteq N_{\text {ess }}$ be the sublattice generated by the roots. This root sublattice decomposes uniquely as a direct sum of simple root lattices $A_{n}(n \geq 1), D_{n}(n \geq 4)$, or $E_{n}(6 \leq n \leq 8)$. These simple factors biject with reducible fibers, each factor being the sublattice of $N_{\text {ess }}$ generated by the components of its reducible fiber that do not meet $s_{0}$. The graph whose vertices are these components, and whose edges are their intersections, is then the $A_{n}, D_{n}$, or $E_{n}$ root diagram; if the identity component and its intersection(s) are included in the graph then the extended root diagram $\tilde{A}_{n}, \tilde{D}_{n}$, or $\tilde{E}_{n}$ results. The quotient group $N_{\text {ess }} / R$ is isomorphic with the Mordell-Weil group of the surface over $\bar{F}(t)$; the isomorphism takes a point $P$ to the projection of the corresponding section $s_{P}$ to $\left\langle s_{0}, f\right\rangle^{\perp}$, and the quadratic form on the Mordell-Weil group induced from the pairing on $N_{\text {ess }}$ is the canonical height. Thus the MordellWeil regulator is $\tau^{2} \operatorname{disc}\left(N_{\text {ess }}\right) / \operatorname{disc}(R)=\tau^{2}|\operatorname{disc}(\operatorname{NS}(S))| / \operatorname{disc}(R)$, where $\tau$ is the size of the torsion subgroup of the Mordell-Weil group.

An elliptic surface has Weierstrass equation $Y^{2}=X^{3}+A(t) X+B(t)$ for polynomials $A, B$ of degrees at most 8, 12 with no common factor of multiplicity at least 4 and 6 respectively, and such that either $\operatorname{deg}(A)>4$ or $\operatorname{deg}(B)>6$ (i.e., such that the condition on common factors holds also at $t=\infty$ when $A, B$ are considered as bivariate homogeneous polynomials of degrees 8,12 ). The reducible fibers then occur at multiple roots of the discriminant $\Delta=-16\left(4 A^{3}+27 B^{2}\right)$ where $B$ does not vanish to order exactly 1 (and at $t=\infty$ if $\operatorname{deg} \Delta \leq 22$ and $\operatorname{deg} B \neq 11$ ). To obtain a smooth model for $S$ we may start from the surface $Y^{2}=X^{3}+A(t) X+B(t)$ in the $\mathbf{P}^{2}$ bundle $\mathbf{P}(O(0) \oplus O(2) \oplus O(3))$ over $\mathbf{P}^{1}$ with coordinates $(1: X: Y)$, and resolve the reducible fibers, as exhibited in Tate's algorithm Ta, which also gives the corresponding Kodaira types and simple root lattices. This information can then be used to calculate the canonical height on the Mordell-Weil group, as in [Si].

The Kummer surface $\operatorname{Km}(A)$ of an abelian surface $A$ is obtained by blowing up the $16=2^{4}$ double points of $A /\{ \pm 1\}$, and is a K3 surface with Picard number $\rho(\operatorname{Km}(A))=\rho(A)+16 \geq 17$. In general $\mathrm{NS}(\operatorname{Km}(A))$ need not consist of divisors defined over $F$, even when $\mathrm{NS}(A)$ does, because each 2-torsion point of $A$ yields a double point of $A /\{ \pm 1\}$ whose blow-up contributes to $\operatorname{NS}(\operatorname{Km}(A))$, and typically $\operatorname{Gal}(\bar{F} / F)$ acts nontrivially on $A[2]$. But when $A$ is principally polarized Dolgachev [Do] constructs another K3 surface $S_{A} / F$, related with $\operatorname{Km}(A)$ by degree-2 maps defined over $\bar{F}$, together with a rank-17 sublattice of $\operatorname{NS}\left(S_{A}\right)$ that is isomorphic with $U \oplus E_{7} \oplus E_{8}$ and consists of divisor classes defined over $F$. It is these surfaces that we parametrize to get at the Shimura curves $\mathrm{X}(N)$.

If $A$ has QM then $\rho(A) \geq 3$, with equality for non-CM surfaces, so $\rho\left(S_{A}\right)=$ $\rho(\operatorname{Km}(A)) \geq 19$. When $A$ has endomorphisms by $\mathcal{O}$, we obtain a sublattice $L_{N} \subseteq \mathrm{NS}\left(S_{A}\right)$ of signature $(1,18)$ and discriminant $2 N$. This even lattice $L_{N}$ is characterized by its signature and discriminant together with the following condition: for each odd $p \mid N$ the dual lattice $L_{N}^{*}$ contains a vector of norm $c / p$ for some $c \in \mathbf{Z}$ such that $\chi_{p}(c)=-\chi_{p}(-2 N / p)$, where $\chi_{p}$ is the Legendre symbol $(\cdot / p)$; equivalently, $N_{\mathrm{ess}}^{*}$ contains a vector of norm $c / p$ with $\chi_{p}(c)=-\chi_{p}(+2 N / p)$. 
There is a corresponding local condition at 2, but it holds automatically once the conditions at all odd $p \mid N$ are satisfied; likewise when $N$ is odd it is enough to check all but one $p \mid N$. The Shimura curve $\mathrm{X}(N) /\left\langle w_{N}\right\rangle$ parametrizes pairs $(S, \iota)$ where $S$ is a K3 surface with $\rho(S) \geq 19$ and $\iota$ is an embedding $L_{N} \hookrightarrow \operatorname{NS}(S)$. If $\rho(S)=20$ then $(S, \iota)$ corresponds to a CM point on $\mathrm{X}(N) /\left\langle w_{N}\right\rangle$ whose discriminant equals disc $(\mathrm{NS}(S))$. The $\mathrm{CM}$ points of discriminant $-N$ or $-4 N$ are the branch points of the double cover $\mathrm{X}(N)$ of $\mathrm{X}(N) /\left\langle w_{N}\right\rangle$. The arithmetic of other $\mathrm{CM}$ points then determines the cover; for instance, if $\mathrm{X}(N) /\left\langle w_{N}\right\rangle$ is rational, we know $\mathrm{X}(N)$ up to quadratic twist, and then a rational CM point of discriminant $D \neq-N,-4 N$ lifts to a pair conjugate over $\mathbf{Q}(\sqrt{-D})$.

The correspondence between $A$ and $S_{A}$ was made explicit by Kumar $\mathrm{Ku}$, Theorem 5.2]. Let $A$ be the Jacobian of a genus-2 curve $C$, and let $I_{2}, I_{4}, I_{6}, I_{10}$ be the Clebsch-Igusa invariants of $C$. (If a principally polarized abelian surface $A$ is not a Jacobian then it is the product of two elliptic curves, and thus cannot have QM unless it is a CM surface.) We give an elliptic model of $S_{A}$ with $N_{\text {ess }}=R=E_{7} \oplus E_{8}$, using a coordinate $t$ on $\mathbf{P}^{1}$ that puts the $E_{7}$ and $E_{8}$ fibers at $t=0$ and $t=\infty$. Any such surface has the formula

$$
Y^{2}=X^{3}+\left(a t^{4}+a^{\prime} t^{3}\right) X+\left(b^{\prime \prime} t^{7}+b t^{6}+b^{\prime} t^{5}\right)
$$

for some $a, a^{\prime}, b, b^{\prime}, b^{\prime \prime}$ with $a^{\prime}, b^{\prime \prime} \neq 0$. (There are five parameters, but the moduli space has dimension only $5-2=3$ as expected, because multiplying $t$ by a nonzero scalar yields an isomorphic surface, and multiplying $a, a^{\prime}$ by $\lambda^{2}$ and $b, b^{\prime}$ by $\lambda^{3}$ for some $\lambda \neq 0$ yields a quadratic twist with the same moduli.) Kumar shows that setting

$$
\left(a, a^{\prime}, b, b^{\prime}, b^{\prime \prime}\right)=\left(-I_{4} / 12,-1,\left(I_{2} I_{4}-3 I_{6}\right) / 108, I_{2} / 24, I_{10} / 4\right)
$$

in (2) yields the surface $S_{J(C)}$. Starting from any surface (2) we may scale $(t, X, Y)$ to $\left(-a^{\prime} t, a^{\prime 2} X, a^{\prime 3} Y\right)$ and divide through by ${a^{\prime}}^{6}$ to obtain an equation of the same form with $a^{\prime}=-1$; doing this and solving (3) for the Clebsch-Igusa invariants $I_{i}$, we find

$$
\left(I_{2}, I_{4}, I_{6}, I_{10}\right)=\left(-24 b^{\prime} / a^{\prime},-12 a, 96 a b^{\prime} / a^{\prime}-36 b,-4 a^{\prime} b^{\prime \prime}\right) .
$$

If $A$ has QM by $\mathcal{O}$, but is not CM, then the elliptic surface (2) has a MordellWeil group of rank 2 and regulator $N$, with each choice of polarization of $A$ corresponding to a different Mordell-Weil lattice. The polarizations for which the map $\mathrm{X}(N) /\left\langle w_{N}\right\rangle \rightarrow \mathcal{A}_{2}$ factors through some $w_{d}$ are those for which the lattice has an involution other than -1 . When this happens, two points on $\mathrm{X}(N) /\left\langle w_{N}\right\rangle$ related by $w_{d}$ yield the same surface (2) but a different choice of Mordell-Weil generators. For example, when $N=6$ and $N=10$ these lattices have Gram matrices $\frac{1}{2}\left(\begin{array}{ll}5 & 1 \\ 1 & 5\end{array}\right)$ and $\frac{1}{2}\left(\begin{array}{ll}8 & 0 \\ 0 & 5\end{array}\right)$ respectively.

Some computational tricks. Often we need elliptic surfaces with an $A_{n}$ fiber for moderately large $n$, that is, for which $4 A^{3}+27 B^{2}$ vanishes to moderately large order $n+1$ at some $t=t_{0}$ at which neither $A$ nor $B$ vanishes. Thus we 
have approximately $(A, B)=\left(-3 a^{2}, 2 a^{3}\right)$ near $t=t_{0}$. Usually one lets $a$ be a polynomial that locally approximates $(-A / 3)^{1 / 2}$ at $t=t_{0}$, and writes

$$
(A, B)=\left(-3\left(a^{2}+2 b\right), 2\left(a^{3}+3 a b\right)+c\right)
$$

for some $b, c$ of valuations $v(b)=\nu, v(c)=2 \nu$ at $t_{0}$. Then $v(\Delta) \geq 2 \nu$ always, and $v(\Delta) \geq 3 \nu$ if and only if $v\left(3 b^{2}-a c\right) \geq 3 \nu$; also if $\mu<\nu$ then $v(\Delta)=2 \nu+\mu$ if and only if $v\left(3 b^{2}-a c\right)=2 \nu+\mu$. See [Ha]; this was also the starting point of our analysis in E2]. For our purposes it is more convenient to allow extended Weierstrass form and write the surface as

$$
Y^{2}=X^{3}+a(t) X^{2}+2 b(t) X+c(t)
$$

with polynomials $a, b, c$ of degrees at most $4,8,12$ such that $(v(b), v(c))=(\nu, 2 \nu)$. Translating $X$ by $-a / 3$ shows that this is equivalent to (5), with $a, b$ divided by 3 (so $\mu=v\left(b^{2}-a c\right)$ in (6) $)$. But (6) tends to produce simpler formulas, both for the surface itself and for the components of the fiber, which are rational if and only if $a$ is a square. For instance, the Shioda-Hall surface with an $A_{18}$ fiber $\mathrm{ShHa}$ can be written simply as

$$
Y^{2}=X^{3}+\left(t^{4}+3 t^{3}+6 t^{2}+7 t+4\right) X^{2}-2\left(t^{3}+2 t^{2}+3 t+2\right) X+\left(t^{2}+t+1\right)
$$

with the $A_{18}$ fiber at infinity, and this is the quadratic twist that makes all of $\mathrm{NS}(S)$ defined over $\mathbf{Q}$. The same applies to $D_{n}$, when $A^{\prime}:=A / t^{2}$ and $B^{\prime}:=B / t^{3}$ are polynomials such that $4 A^{\prime 3}+27 B^{\prime 2}$ has valuation $n-4$. See for instance (19) below. When we want singular fibers at several $t$ values we use an extended Weierstrass form (6) for which $(v(b), v(c))=(\nu, 2 \nu)$ holds (possibly with different $\nu$ ) at each of these $t$.

Having parametrized our elliptic surface $S$ with $L_{N} \hookrightarrow \operatorname{NS}(S)$, we seek specializations of rank 20 to locate CM points. In all but finitely many cases $S$ has an extra Mordell-Weil generator. In the exceptional cases, either some of the reducible fibers merge, or one of those fibers becomes more singular, or there is an extra $A_{1}$ fiber. Such CM points are easy to locate, though some mergers require renormalization to obtain a smooth model and find the CM discriminant $D$, as we shall see. When there is an extra Mordell-Weil generator, its height is at least $|D| / 2 N$, but usually not much larger. (Equality holds if and only if the extra generator is orthogonal to the generic Mordell-Weil lattice; in particular this happens if $S$ has generic Mordell-Weil rank zero.) The larger the height of the extra generator, the harder it typically is to find the surface. This has the curious consequence that while the difficulty of parametrizing $S$ increases with $N$, the $\mathrm{CM}$ points actually become easier to find. In some cases we cannot solve for the coefficients directly. We thus adapt the methods of [E3], exhaustively searching for a solution modulo a small prime $p$ and then lifting it to a $p$-adic solution to enough accuracy to recognize the underlying rational numbers. We choose the smallest $p$ such that $\chi_{p}(-D)=+1$, so that reduction $\bmod p$ does not raise the Picard number, and we can save a factor of $p$ in the exhaustive search by first counting points mod $p$ on each candidate $S$ to identify the one with the correct CM. 
For large $N$ we use the following variation of the $p$-adic lifting method to find the Shimura curve $\mathrm{X}(N) /\left\langle w_{N}\right\rangle$ and the surfaces $S$ parametrized by it. First choose some indefinite primitive sublattice $L^{\prime} \subset L_{N}$ and parametrize all $S$ with $\operatorname{NS}(S) \supseteq L^{\prime}$. Search in that family modulo a small prime $p$ to find a surface $S_{0}$ with the desired $L_{N}$. Let $f_{1}, f_{2}$ be simple rational functions on the $\left(S, L^{\prime}\right)$ moduli space. We hope that the degrees, call them $d_{i}$, of the restriction of $f_{i}$ to $\mathrm{X}(N) /\left\langle w_{N}\right\rangle$ are positive but small; that $f_{1}$ is locally $1: 1$ on the point of $\mathrm{X}(N) /\left\langle w_{N}\right\rangle$ parametrizing $S_{0}$; and that the map $\left(f_{1}, f_{2}\right): \mathrm{X}(N) /\left\langle w_{N}\right\rangle \rightarrow \mathbf{A}^{2}$ is generically $1: 1$ to its image in the affine plane. For various small lifts $\tilde{f}_{1}$ of $f_{1}\left(S_{0}\right)$ to $\mathbf{Q}$, lift $S_{0}$ to a surface $S / \mathbf{Q}_{p}$ with $f_{1}(S)=\tilde{f}_{1}$, compute $f_{2}(S)$ to high $p$-adic precision, and use lattice reduction to recognize $f_{2}(S)$ as the solution of a polynomial equation $F\left(f_{2}\right)=0$ of degree (at most) $d_{1}$. Discard the few cases where the degree is not maximal, and solve simultaneous linear equations to guess the coefficients of $F$ as polynomials of degree at most $d_{2}$ in $\tilde{f}_{1}$. At this point we have a birational model $F\left(f_{1}, f_{2}\right)=0$ for $\mathrm{X}(N) /\left\langle w_{N}\right\rangle$. Then recover a smooth model of the curve (using Magma if necessary), recognize the remaining coefficients of $S$ as rational functions by solving a few more linear equations, and verify that the surface has the desired embedding $L_{N} \hookrightarrow \operatorname{NS}(S)$.

\section{$3 \quad N=6$ : The first Shimura curve}

The K3 surfaces. We take $N_{\text {ess }}=R=A_{2} \oplus D_{7} \oplus E_{8}$, which has discriminant $3 \cdot 4 \cdot 1=12=2 N$, and the correct behavior at 3 because $A_{2}^{*}$ contains vectors of norm $2 / 3$ with $\chi_{3}(2)=-\chi_{3}(2 \cdot 6 / 3)[=-1]$. We choose the rational coordinate $t$ on $\mathbf{P}^{1}$ such that the $A_{2}, D_{7}$, and $E_{8}$ fibers are at $t=1,0$, and $\infty$ respectively. If we relax the condition at $t=1$ by asking only that the discriminant vanish to order at least 2 rather than 3 then the general such surface can be written as

$$
Y^{2}=X^{3}+\left(a_{0}+a_{1} t\right) t X^{2}+2 a_{0} b t^{3}(t-1) X+a_{0} b^{2} t^{5}(t-1)^{2}
$$

for some $a_{0}, a_{1}, b$, with $a_{1} b \neq 0$ lest the surface be too singular at $t=0$. The discriminant is then $t^{9}(t-1)^{2} \Delta_{1}(t)$ with $\Delta_{1}$ a cubic polynomial such that $\Delta(1)=$ $-64 a_{0} a_{1}\left(a_{0}+a_{1}\right)^{2} b^{2}$. Thus $\Delta_{1}(1)=0$ if and only if $a_{1}=0$ or $a_{0}+a_{1}=0$. In the latter case the surface has additive reduction at $t=1$. Hence we must have $a_{1}=0$. The non-identity components of the resulting $A_{2}$ fiber at $t=1$ then have $X=O(t-1)$; we calculate that $X=x_{1}(t-1)+O\left((t-1)^{2}\right)$ makes $Y^{2}=\left(x_{1}+b\right)^{2} a_{0}(t-1)^{2}+O(t-1)^{3}$. Therefore these components are rational if and only if $a_{0}$ is a square. We can then replace $(X, Y, b)$ by $\left(a_{0} X, a_{0}^{3 / 2} Y, a_{0} b\right)$ in (17) to obtain the formula

$$
Y^{2}=X^{3}+t X^{2}+2 b t^{3}(t-1) X+b^{2} t^{5}(t-1)^{2}
$$

for the general elliptic K3 surface with $N_{\text {ess }}=R=A_{2} D_{7} E_{8}$ and rational $A_{2}$ components. The two components of the $D_{7}$ fiber farthest from the identity component then have $X=b t^{2}+O\left(t^{3}\right)$, so $Y^{2}=b^{3} t^{6}+O\left(t^{7}\right)$; thus these components are both rational as well if and only $b$ is a square, say $b=r^{2}$. Then $b$ and $r$ 
are rational coordinates on the Shimura curves $\mathrm{X}(6) /\left\langle w_{2}, w_{3}\right\rangle$ and $\mathrm{X}(6) /\left\langle w_{6}\right\rangle$ respectively, with the involution $w_{2}=w_{3}$ on $\mathrm{X}(6) /\left\langle w_{6}\right\rangle$ taking $r$ to $-r$.

The elliptic surface (8) has discriminant $\Delta=16 b^{3} t^{9}(t-1)^{3}\left(27 b\left(t^{2}-t\right)-4\right)$. Thus the formula (8) fails at $b=0$, and also of course at $b=\infty$. Near each of these two points we change variables to obtain a formula that extends smoothly to $b=0$ or $b=\infty$ as well. These formulas require extracting respectively a fourth and third root of $\beta$, presumably because $b=0$ and $b=\infty$ are elliptic points of the Shimura curve. For small $b$, we take $b=\beta^{4}$ and replace $(t, X, Y)$ by $\left(t / \beta^{2}, X / \beta^{2}, Y / \beta^{3}\right)$ to obtain

$$
Y^{2}=X^{3}+t X^{2}+2 t^{3}\left(t-\beta^{2}\right) X+t^{5}\left(t-\beta^{2}\right)^{2},
$$

with the $A_{2}$ fiber at $t=\beta^{2}$ rather than $t=1$. When $\beta=0$, this fiber merges with the $D_{7}$ fiber at $t=0$ to form a $D_{10}$ fiber, but we still have a K3 surface, namely $Y^{2}=X^{3}+t X^{2}+2 t^{4} X+t^{7}$, with $L=R=D_{10} \oplus E_{8}$. This is the CM point of discriminant -4 . For large $b$, we write $b=1 / \beta^{3}$ and replace $(X, Y)$ by $\left(X / \beta^{2}, Y / \beta^{3}\right)$ to obtain

$$
Y^{2}=X^{3}+\beta^{2} t X^{2}+2 \beta t^{3}(t-1) X+t^{5}(t-1)^{2}
$$

then taking $\beta \rightarrow 0$ yields the surface $Y^{2}=X^{3}+t^{5}(t-1)^{2}$ with $N_{\text {ess }}=R_{0}=$ $A_{2} \oplus E_{8} \oplus E_{8}$ : the $t=0$ fiber changes from $D_{7}$ to $E_{8}$, and the $t=1$ fiber becomes additive but still contributes $A_{2}$ to $R$ (Kodaira type IV rather than $\mathrm{I}_{3}$ ). This is the CM point of discriminant -3 .

Two more CM points. The factor $27 b\left(t^{2}-t\right)-4$ of $\Delta$ is a quadratic polynomial in $t$ of discriminant $27 b(27 b+16)$. Hence at $b=-16 / 27$ we have $N_{\text {ess }}=R=A_{1} \oplus A_{2} \oplus D_{7} \oplus E_{8}$, and we have located the CM point of discriminant -24 . Three points fix a rational coordinate on $\mathbf{P}^{1}$, so we can compare with the coordinate used in [E1, Table 1], which puts the CM points of discriminant -3 , -4 , and -24 at $\infty, 1$, and 0 respectively; thus that coordinate is $1+27 b / 16$. This also confirms that $\mathrm{X}(6)$ is obtained by extracting a square root of $-\left(27 r^{2}+16\right)$.

We next locate a CM point of discriminant -19 by finding $b$ for which the surface (8) has a section $s_{P}$ of canonical height 19/12. This is the smallest possible canonical height for a surface with $R=A_{2} \oplus D_{7} \oplus E_{8}$, because the naïve height is at least 4 and the height corrections at the $A_{2}$ and $D_{7}$ fibers can reduce it by at most $2 / 3$ and $7 / 4$ respectively, reaching $4-2 / 3-7 / 4=19 / 12$. Let $(X(t), Y(t))$ be the coordinates of a point $P$ of height $19 / 12$. Then $X(t)$ and $Y(t)$ are polynomials of degree at most 4 and 6 respectively (else $s_{P}$ intersects $s_{0}$ and the naïve height exceeds 4 ), and $X$ vanishes at $t=1$ (so $s_{P}$ passes through a non-identity component of the $A_{2}$ fiber) and has the form $b t^{2}+O\left(t^{3}\right)$ at $t=0$ (so $s_{P}$ meets one of the components of the $D_{7}$ fiber farthest from the identity component). That is, $X=b\left(t^{2}-t^{3}\right)\left(1+t_{1} t\right)$ for some $t_{1}$. Substituting this into (8) and dividing by the known square factor $\left(t^{4}-t^{3}\right)^{2}$ yields $b^{3}$ times

$$
-t_{1}^{3} t^{4}+\left(t_{1}^{3}-3 t_{1}^{2}\right) t^{3}+3\left(t_{1}^{2}-t_{1}\right) t^{2}+\left(\left(3 t_{1}-1\right)+b^{-1} t_{1}^{2}\right) t+1
$$

so we seek $b, t_{1}$ such that the quartic (11) is a square. We expand its square root in a Taylor expansion about $t=0$ and set the $t^{3}$ and $t^{4}$ coefficients equal to 
zero. This gives a pair of polynomial equations in $b$ and $t_{1}$, which we solve by taking a resultant with respect to $t_{1}$. Eliminating a spurious multiple solution at $b=0$, we finally obtain $\left(b, t_{1}\right)=(81 / 64,-9)$, and confirm that this makes (11) a square, namely $\left(27 t^{2}-18 t-1\right)^{2}$. Therefore $81 / 64$ is the $b$-coordinate of a CM point of discriminant -19 . Then $1+27 b / 16=3211 / 2^{10}$, same as the value obtained in [E1].

Clebsch-Igusa coordinates. The next diagram shows the graph whose vertices are the zero-section (circled) and components of reducible fibers of an elliptic K3 surface $S$ with $N_{\text {ess }}=A_{2} \oplus D_{7} \oplus E_{8}$, and whose edges are intersections between pairs of these rational curves on the surface. Eight of the vertices form an extended root diagram of type $\tilde{E}_{7}$, and are marked with their multiplicities in a reducible fiber of type $E_{7}$ of an alternative elliptic model for $S$. We may take either of the unmarked vertices of the $\tilde{D}_{7}$ subgraph as the zero-section. Then the essential lattice of the new model includes an $E_{8}$ root diagram as well as the forced $E_{7}$. We can thus apply Kumar's formulas to this model once we compute its coefficients.

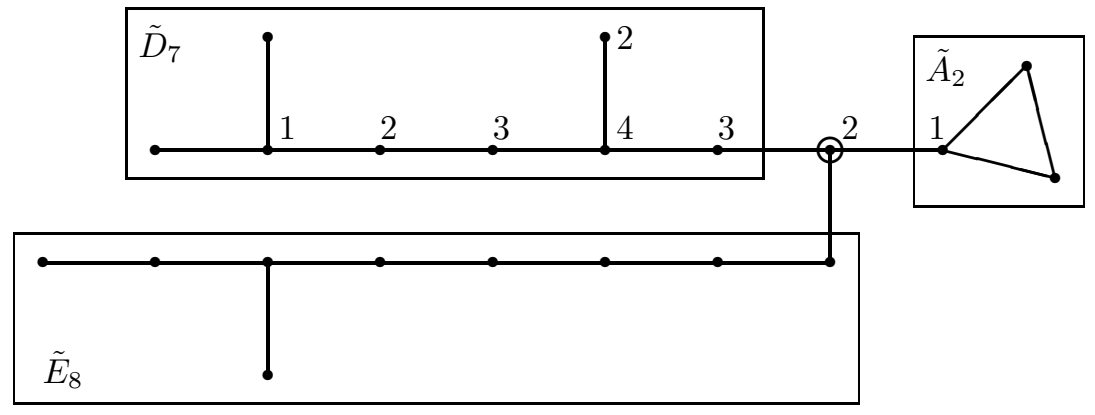

Figure 1: An $\tilde{E}_{7}$ divisor supported on the zero-section and fiber components of an $A_{2} D_{7} E_{8}$ surface

The sections of the $\tilde{E}_{7}$ divisor are generated by 1 and $u:=X /\left(t^{4}-t^{3}\right)+b / t$. Thus $u: S \rightarrow \mathbf{P}^{1}$ gives the new elliptic fibration. Taking $X=\left(t^{3}-t^{2}\right)(t u-b)$ in (8) and dividing by $\left(t^{4}-t^{3}\right)^{2}$ yields $Y_{1}^{2}=Q(t)$ for some quartic $Q$. Using standard formulas for the Jacobian of such a curve, and bringing the resulting surface into Weierstrass form, we obtain a formula (2) with $\left(a, a^{\prime}, b, b^{\prime}, b^{\prime \prime}\right)$ replaced by $\left(-3 b, 1,-2 b^{2},-(b+1),-b^{3}\right)$. As expected this surface has Mordell-Weil rank 2 with generators of height $5 / 2$, namely

$\left(r^{6} t^{4}+2\left(r^{4}+r^{3}\right) t^{3}+\left(r^{2}+1\right) t^{2}, r^{9} t^{6}+3\left(r^{7}+r^{6}\right) t^{5}+3\left(r^{5}+r^{4}+r^{3}\right) t^{4}+\left(r^{3}+1\right) t^{3}\right)$

and the image of this section under $r \leftrightarrow-r$ (recall that $b=r^{2}$ ). The formula (4) yields the Clebsch-Igusa coordinates

$$
\left(I_{2}, I_{4}, I_{6}, I_{10}\right)=\left((24 b+1), 36 b, 72 b(5 b+4), 4 b^{3}\right) .
$$




\section{$4 \quad N=14:$ The CM point of discriminant -67}

The K3 surfaces. Here we take $N_{\text {ess }}=R=A_{3} \oplus A_{6} \oplus E_{8}$, which has discriminant $4 \cdot 7 \cdot 1=28=2 N$, and the correct behavior at 7 because $A_{6}^{*}$ contains vectors of norm $6 / 7$ with $\chi_{7}(6)=-\chi_{7}(2 \cdot 14 / 7)[=-1]$. We put the $A_{3}, A_{6}$, and $E_{8}$ fibers at $t=1,0$, and $\infty$ respectively. We then seek an extended Weierstrass form (6) with $a, b, c$ of degrees $2,4,7$ such that $t^{3}-t^{2}\left|b,\left(t^{3}-t^{2}\right)^{2}\right| c$, and $\left(t^{3}-t^{2}\right)^{6} \mid b^{2}-a c$. This gives at least $A_{3}, A_{5}, E_{8}$. It is then easy to impose the extra condition $t^{7} \mid \Delta$, and we obtain $a=\lambda\left((s+1) t^{2}+\left(3 s^{2}+2 s\right) t+s^{3}\right)$, $b=\lambda^{2}(s+1)\left((4 s+2) t+2 s^{2}\right)\left(t^{3}-t^{2}\right), c=\lambda^{3}(s+1)^{2}(t+s)\left(t^{3}-t^{2}\right)^{2}$ for some $s, \lambda$. The twist $\lambda$ must be chosen so that $a(0)$ and $a(1)$ are both squares; this is possible if and only if $s^{2}+s$ is a square, so $s=r^{2} /(2 r+1)$ for some $r$. Thus $r$ and $s$ are rational coordinates on $\mathrm{X}(14) /\left\langle w_{14}\right\rangle$ and $\mathrm{X}(14) /\left\langle w_{2}, w_{7}\right\rangle$ respectively, with the involution $w_{2}=w_{7}$ on $\mathrm{X}(14) /\left\langle w_{14}\right\rangle$ taking $r$ to $-r /(2 r+1)$. The formula in terms of $r$ is cleaner if we let the $A_{3}$ fiber move from $t=1$; putting it at $t=2 r+1$ yields

$$
\begin{aligned}
& \left.a=\left((r+1)^{2}\right) t^{2}+\left(3 r^{4}+4 r^{3}+2 r^{2}\right) t+r^{6}\right), \\
& b=2(r+1)^{2}\left(\left(2 r^{2}+2 r+1\right) t+r^{4}\right)(t-(2 r+1)) t^{2}, \\
& c=(r+1)^{4}(t-(2 r+1))^{2}\left(t+r^{2}\right) t^{4} .
\end{aligned}
$$

Easy $\mathrm{CM}$ points. At $r=0$, the $A_{6}$ fiber becomes $E_{7}$, so we have a CM point with $D=-8$; at $r=-1 / 2$, the $A_{3}$ and $A_{6}$ fibers merge to $A_{10}$, giving a CM point with $D=-11$. These have $s=0, s=\infty$ respectively. There is an extra $A_{1}$ fiber when $11 s^{2}+3 s+8=0$; the roots of this irreducible quadratic give the CM points with $D=-56$ (and their lifts to $\mathrm{X}(14) /\left\langle w_{14}\right\rangle$ are the branch points of the double cover $\mathrm{X}(14))$. In [E1] we gave a rational coordinate $t$ on $\mathrm{X}(14) /\left\langle w_{2}, w_{7}\right\rangle$ for which the CM points of discriminants $-8,-11$, and -56 had $t=0, t=-1$, and $16 t^{2}+13 t+8=0$ respectively. Therefore that $t$ is our $-s /(s+1)$.

A harder CM point. At the CM point of discriminant -67 our surface has a section of height $67 / 28=4-(3 / 4)-(6 / 7)$. Thus $Y^{2}=X^{3}+a X^{2}+b X+c$ has a solution in polynomials $X, Y$ of degrees 4,6 with $X(0)=X(2 h+1)=0$ and $Y$ having valuation exactly 1 at $t=0$ and $t=2 h+1$. An exhaustive search mod 17 quickly finds an example, whose lift to $\mathbf{Q}_{17}$ then yields $r=-35 / 44$ with

$$
X=\frac{3^{4}}{5^{2} 22^{5}} t(22 t+13)\left(527076 t^{2}+760364 t+275625\right) .
$$

Thus $s=-1225 / 1144$, and $-s /(s+1)$ confirms the entry $-1225 / 81$ in the $|D|=67$ row of [E1, Table 5].

\section{$5 \quad N=57$ : The first curve $\mathrm{X}(N) / w_{N}$ of positive genus}

The K3 surfaces. We cannot have $N_{\text {ess }}=R$ here because there is no root lattice of rank 17 and discriminant $6 \cdot 19$. Instead we take for $R$ the rank-16 lattice 
$A_{5} \oplus A_{11}$ of discriminant $6 \cdot 12=72$, and require an infinite cyclic Mordell-Weil group $N_{\text {ess }} / R$ with a generator corresponding to a section that meets the $A_{5}$ and $A_{11}$ fibers in non-identity components farthest from the $A_{5}$ identity and nearest the $A_{11}$ identity respectively, and does not meet the zero-section (i.e., for which $X$ is a polynomial of degree at most 4 in $t$ ). Such a point has canonical height

$$
4-\frac{3 \cdot 3}{6}-\frac{1 \cdot 11}{12}=\frac{19}{12}=\frac{2 N}{\operatorname{disc} R} .
$$

Thus $\operatorname{disc}\left(N_{\text {ess }}\right)$ has the desired discriminant $2 N$. We may check the local conditions by noting that $A_{5}^{*}$ contains a vector of norm $4 / 3$ that remains in $N_{\text {ess }}^{*}$, and $\chi_{3}(4)=-\chi_{3}(2 \cdot 57 / 3)[=+1]$. We put the $A_{5}$ fiber at $t=0$ and the $A_{11}$ fiber at $t=\infty$. We eventually obtain the following parametrization in terms of a coordinate $r$ on the rational curve $\mathrm{X}(57) /\left\langle w_{3}, w_{19}\right\rangle$ : let

$$
\begin{aligned}
p(r) & =4(r-1)\left(r^{2}-2\right)+1, \\
d & =\left(r^{2}-1\right)^{2}(9 t+(2 r-1) p(r)), \\
c & =9 t^{2}-(2 r-1)\left(8 r^{2}+4 r-22\right) t+(2 r-1)^{2} p(r), \\
b & =\left(t-\left(r^{2}-2 r\right)\right) c+d, \\
a & =\left(t-\left(r^{2}-2 r\right)\right)^{2} c+2\left(t-\left(r^{2}-2 r\right)\right) d+\left(r^{2}-1\right)^{4}((4 r+4) t+p(r)) ;
\end{aligned}
$$

Then the surface is

$$
Y^{2}=X^{3}+a X^{2}+8(r-1)^{4}(r+1)^{5} b t^{2} X+16(r-1)^{8}(r+1)^{10} c t^{4},
$$

with a section of height 19/12 at

$$
X=-\frac{4(r-1)^{4}(r+1)^{5}(2 r-1) t^{2}}{\left(r^{2}-r+1\right)^{2}}+\frac{4(r-2)(r+1)^{4} t^{3}}{r^{2}-r+1} .
$$

The components of the $A_{11}$ fiber are rational because the leading coefficient of $a$ is 9 , a square; the constant coefficient is $\left(r^{2}-r+1\right)^{4} p(r)$, so $\mathrm{X}(57) /\left\langle w_{57}\right\rangle$ is obtained by extracting a square root of $p(r)$. This gives the elliptic curve with coefficients $\left[a_{1}, a_{2}, a_{3}, a_{4}, a_{6}\right]=[0,-1,1,-2,2]$, whose conductor is 57 as expected (see e.g. Cremona's tables $\mathrm{Cr}$ where this curve appears as 57-A1(E)).

This curve has rank 1 , with generator $P=(2,1)$. The point at infinity is the CM point of discriminant -19 ; this may be seen by substituting $1 / s$ for $r$ and $\left(t / s^{3}, X / s^{12}, Y / s^{18}\right)$ for $(t, X, Y)$, then letting $s \rightarrow 0$ to obtain the surface

$$
Y^{2}=X^{3}+\left(9 t^{4}-16 t^{3}+4 t\right) X^{2}+\left(72 t^{5}-128 t^{4}\right) X+\left(144 t^{6}-256 t^{5}\right)
$$

with a $D_{6}$ fiber at $t=0$ rather than an $A_{5}$. Then we still have a section $(X, Y)=$ $\left(4 t^{3}-8 t^{2},(3 t-5)\left(t^{4}-t^{3}\right)\right)$ of height $19 / 12$, but there is a 2 -torsion point $(X, Y)=$ $(-4 t, 0)$ so $\operatorname{disc}\left(N_{\text {ess }}\right)=-\operatorname{disc}(\mathrm{NS}(S))$ drops to $4 \cdot 12 \cdot(19 / 12) / 2^{2}=19$. The remaining rational $\mathrm{CM}$ points on $\mathrm{X}(57) /\left\langle w_{57}\right\rangle$ come in six pairs $\pm n P$ :

\begin{tabular}{c||c|c|c|c|c|c}
$n$ & 1 & 2 & 3 & 4 & 5 & 8 \\
\hline$r$ & 2 & 1 & -1 & 0 & $5 / 4$ & $13 / 9$ \\
\hline$-D$ & 7 & 4 & 16 & 28 & 43 & 163
\end{tabular}


The last three of these have extra sections $X=-4 t, X=0$, and

$$
X=-28 \cdot 11^{3}\left(\left(t^{2} / 3^{6}\right)+\left(415454 t / 3^{18}\right)\right)
$$

respectively. At $r=2$, the $A_{11}$ fiber becomes an $A_{12}$ and our generic MordellWeil generator becomes divisible by 3 ; the new generator $\left(-972 t, 26244 t^{2}\right)$ has height $4-(5 / 6)-(40 / 13)=7 / 78$, so $\operatorname{disc} N_{\text {ess }}=6 \cdot 13 \cdot(7 / 78)=7$. At $r=1$, the $A_{5}$ and $A_{11}$ fibers together with the section all merge to form a $D_{18}$ fiber: let $r=1+s$ and change $(t, X)$ to $\left(s t-1,-8 s^{3} X\right)$, divide by $(-2 s)^{9}$, and let $s \rightarrow 0$ to obtain the second Shioda-Hall surface

$$
X^{3}+\left(t^{3}+8 t\right) X^{2}-\left(32 t^{2}+128\right) X+256 t
$$

with a $D_{18}$ fiber at $t=\infty$ ShHa. At $t=-1$, the reducible fibers again merge, this time forming an $A_{17}$ while the Mordell-Weil generator's height drops to $4-(4 \cdot 14 / 18)=8 / 9$, whence $\operatorname{disc}\left(N_{\text {ess }}\right)=16$.

We find four more rational $\mathrm{CM}$ values of $r$ that do not lift to rational points on $\mathrm{X}(57) /\left\langle w_{57}\right\rangle$, namely $r=5,1 / 2,17 / 16,-7 / 4$, for discriminants $-123,-24$, -267 , and $-627=-11.57$ respectively. The first of these again has an $A_{12}$ fiber, this time with the section of height $4-(9 / 6)-(12 / 13)=41 / 26$; the second has a rational section at $X=0$; in the remaining two cases we find the extra section by $p$-adic search:

$$
X=-\frac{11^{3} 3^{2}}{2^{21} 91^{2}} t^{2}\left(7840 t^{2}-2037 t+3267\right)
$$

for $r=17 / 16$, and

$$
X=\frac{3^{5} 11^{4} t^{2} q(t)}{2^{12}\left(81920 t^{3}+9216 t^{2}+23868 t+39339\right)^{2}}
$$

for $r=-7 / 4$, where $q(t)$ is the quintic

$$
\begin{aligned}
& 419430400 t^{5}+2846883840 t^{4}+17148174336 t^{3} \\
& +78784560576 t^{2}+175272616341 t-12882888 .
\end{aligned}
$$

Using $\mathrm{Ar}$ we can show that there are no further rational CM values.

\section{$6 \quad N=206:$ The last curve $\mathrm{X}(N) / w_{N}$ of genus zero}

Summary of results. Again we take $N_{\text {ess }}$ of rank 16 and an infinite cyclic Mordell-Weil group, here $R=A_{2} \oplus A_{4} \oplus A_{10}$ with a Mordell-Weil generator of height $412 / 165=6-(1 \cdot 2 / 3)-(2 \cdot 3) / 5-(2 \cdot 9) / 11$. With the reducible fibers placed at $1,0, \infty$ as usual, the choice of $R$ means $\Delta=t^{5}(t-1)^{3} \Delta_{1}$ with $\Delta_{1}$ of degree $24-(3+5+11)=5$ and $\Delta_{1}(0), \Delta_{1}(1) \neq 0$; the generator must then have $X(t)=X_{1}(t) /\left(t-t_{0}\right)^{2}$ for some sextic $X_{1}$ and some $t_{0} \neq 0,1$, with the corresponding section passing through a non-identity component of the $A_{2}$ fiber and 
components at distance 2 from the identity of $A_{4}$ and $A_{10}$. We eventually succeed in parametrizing such surfaces, finding a rational coordinate on the modular curve $\mathrm{X}(206) /\left\langle w_{206}\right\rangle$. These elliptic models do not readily exhibit the involution $w_{2}=w_{103}$ on this curve, so we recover this involution from the fact that it must permute the branch points of the double cover $\mathrm{X}(206)$ of $\mathrm{X}(206) /\left\langle w_{206}\right\rangle$. We locate these branch points as simple zeros of the discriminant of $\Delta_{1}$. As expected, there are 20 (this is the class number of $\mathbf{Q}(\sqrt{-206})$ ), forming a single Galois orbit. We find a unique involution of the projective line $\mathrm{X}(206) /\left\langle w_{206}\right\rangle$ that permutes these zeros. This involution has two fixed points, so we switch to a rational coordinate $r$ on $\mathrm{X}(206) /\left\langle w_{206}\right\rangle$ that makes the involution $r \leftrightarrow-r$. Then $r_{0}:=r^{2}$ is a rational coordinate on $\mathrm{X}(206) /\left\langle w_{2}, w_{103}\right\rangle$, and the 20 branch points are the roots of $P_{10}\left(r^{2}\right)$ where $P_{10}$ is the degree-10 polynomial

$$
\begin{aligned}
P_{10}\left(r_{0}\right)=8 r_{0}^{10}-13 r_{0}^{9}-42 r_{0}^{8}-331 r_{0}^{7}-220 r_{0}^{6}+733 r_{0}^{5} \\
+6646 r_{0}^{4}+19883 r_{0}^{3}+28840 r_{0}^{2}+18224 r_{0}+4096 .
\end{aligned}
$$

As a further check on the computation, $P_{10}$ has dihedral Galois group, discriminant $-2^{138} 103^{7}$, and field discriminant $-2^{12} 103^{5}$, while $P_{10}\left(r^{2}\right)$ has discriminant $2^{311} 103^{14}$ and field discriminant $2^{27} 103^{10}$. We find that $r=0, \pm 1, \pm 2, \infty$ give CM points of discriminants $D=-4,-19,-163,-8$ respectively; evaluating $P_{10}\left(r^{2}\right)$ at any of these points gives $-D$ times a square, showing that the Shimura curve $\mathrm{X}(206)$ has the equation $s^{2}=-P_{10}\left(r^{2}\right)$ over $\mathbf{Q}$. The curves $\mathrm{X}(206) /\left\langle w_{2}\right\rangle$, $\mathrm{X}(206) /\left\langle w_{103}\right\rangle$ are then the double covers $s_{0}^{2}=-P_{10}\left(r_{0}\right), s_{0}^{\prime 2}=-r_{0} P_{10}\left(r_{0}\right)$ of the $r_{0}$-line $\mathrm{X}(206) /\left\langle w_{2}, w_{103}\right\rangle$ (in that order, because $w_{103}$ cannot fix a CM point of discriminant -4 or -8$)$.

\section{Acknowledgements}

I thank Benedict H. Gross, Joseph Harris, John Voight, Abhinav Kumar, and Matthias Schütt for enlightening discussion and correspondence, and for several references concerning Shimura curves and K3 surfaces. I thank M. Schütt, Jeechul Woo, and the referees for carefully reading an earlier version of the paper and suggesting many corrections and improvements. The symbolic and numerical computations reported here were carried out using the packages GP, MAXIMA, and Magma.

\section{References}

Ar. Arno, S.: The Imaginary Quadratic Fields of Class Number 4, Acta Arith. 40 (1992), 321-334.

BHPV. Barth, W.P., Hulek, K., Peters, C.A.M., and van de Ven, A.: Compact Complex Surfaces (2nd ed.). Berlin: Springer, 2004.

Cr. Cremona, J.E.: Algorithms for Modular Elliptic Curves. Cambridge University Press, 1992; 2nd edition, 1997. Book and data electronically available at http://www.warwick.ac.uk/staff/J.E.Cremona/book/fulltext/index.html .

Do. Dolgachev, I.: appendix to F. Galluzzi and G. Lombardo, Correspondences between K3 surfaces, Michigan Math. J. 52 (2004) \#2, 267-277. 
E1. Elkies, N.D.: Shimura curve computations, pages 1-47 in Algorithmic Number Theory (Portland, OR, 1998; J. Buhler, ed.; Lect. Notes in Computer Sci. \#1423; Berlin: Springer, 1998) = http://arXiv.org/abs/math/0005160 .

E2. Elkies, N.D.: Rational points near curves and small nonzero $\left|x^{3}-y^{2}\right|$ via lattice reduction, pages 33-63 in Algorithmic Number Theory (Leiden, 2000; W. Bosma, ed.; Lect. Notes in Computer Sci. \#1838; Berlin: Springer, 2000) $=$ http://arXiv.org/abs/math/0005139.

E3. Elkies, N.D.: Shimura curves for level-3 subgroups of the $(2,3,7)$ triangle group, and some other examples, pages 302-316 in Algorithmic Number Theory (Berlin, 2006; F. Hess, S. Pauli, and M. Pohst, ed.; Lect. Notes in Computer Sci. \#4076; Berlin: Springer, 2006) = http://arXiv.org/abs/math/0409020 .

E4. Elkies, N.D.: Three lectures on elliptic surfaces and curves of high rank, Oberwolfach lecture notes $2007=$ http://arXiv.org/abs/0709.2908 .

Er. $\quad$ Errthum, E.: Singular Moduli of Shimura Curves. Ph.D. thesis, Univ. of Maryland, $2007=$ http://arxiv.org/abs/0711.4316 .

GR. González, J., and Rotger, V.: Equations of Shimura curves of genus two, International Math. Research Notices 14 (2004), 661-674.

GZ. Gross, B.H., and Zagier, D.: On singular moduli, J. für die reine und angew. Math. 335 (1985), 191-220.

Ha. Hall, M.: The Diophantine equation $x^{3}-y^{2}=k$. Pages 173-198 in Computers in Number Theory (A.Atkin, B.Birch, eds.; Academic Press, 1971).

HM. Hashimoto, K.-i., and Murabayashi, N.: Shimura curves as intersections of Humbert surfaces and defining equations of QM-curves of genus two, Tohoku Math. Journal (2) 47 (1995), \#2, 271-296.

Ku. Kumar, A.: K3 Surfaces of High Rank. Ph.D. thesis, Harvard, 2006.

LY. Lian, B.H., Yau, S.-T.: Mirror Maps, Modular Relations and Hypergeometric Series I. Preprint, 1995 (http://arXiv.org/abs/hep-th/9507151).

PSS. Piateckii-Shapiro, I., and Šafarevič, I. R.: A Torelli theorem for algebraic surfaces of type K3 [Russian], Izv. Akad. Nauk SSSR, Ser. Mat. 35 (1971), 530572 .

Rob. Roberts, D.P.: Shimura curves analogous to $\mathrm{X}_{0}(N)$. Ph.D. thesis, Harvard, 1989.

Rot1. Rotger, V.: Shimura curves embedded in Igusa's threefold. Pages 263-276 in Modular curves and abelian varieties (J. Cremona, J.-C. Lario, J. Quer, and K. Ribet, eds.; Progress in Math. 224), Basel: Birkhäuser, $2004=$ http://arXiv.org/abs/math/0312435 .

Rot2. Rotger, V.: Modular Shimura varieties and forgetful maps, Trans. Amer. Math. Soc. 356 (2004), 1535-1550 = http://arXiv.org/abs/math/0303163 .

Sh. Shioda, T.: The elliptic K3 surfaces with a maximal singular fibre, C. R. Acad. Sci. Paris Ser. I 337 (2003), 461-466.

Si. Silverman, J.H.: Computing Heights on Elliptic Curves, Math. of Computation 51 \#183 (1988), 339-358.

Ta. Tate, J.: Algorithm for determining the type of a singular fiber in an elliptic pencil. Pages 33-52 in Modular Functions of One Variable IV (Antwerp, 1972; B.J. Birch and W. Kuyk, eds.); Lect. Notes in Math. \#476, Berlin: Springer, 1975.

Vi. Vignéras, M.-F.: Arithmétique des Algèbres de Quaternions. Berlin: Springer, 1980 (SLN 800).

Wa. Watkins, M.: Class numbers of imaginary quadratic fields, Math. of Computation 73 (2003), 907-938. 